\title{
Incidence of Hodgkin lymphoma in HIV-positive and HIV-negative patients at a tertiary hospital in South Africa (2005 - 2016) and comparison with other African countries
}

\author{
N Naidoo, ${ }^{1}$ MB BCh, MPH; A Abayomi, ${ }^{1}$ MBBS, MRCP, FCPath, MPhil, DHSM; C Locketz, ${ }^{2}$ MB ChB, FC Path (Anat), MMed (Anat Path); \\ F Musaigwa, ${ }^{1}$ MPath (Haem Path); R Grewal, ${ }^{1}$ MB ChB, FC Path (Haem), MMed (Haem Path) \\ ${ }^{1}$ Division of Haematopathology, Department of Pathology, National Health Laboratory Service and Stellenbosch University, Cape Town, South Africa \\ ${ }^{2}$ Division of Anatomical Pathology, Department of Pathology, National Health Laboratory Service and Stellenbosch University, Cape Town, \\ South Africa
}

Corresponding author: N Naidoo (nasheen.naidoo@nhls.ac.za)

\begin{abstract}
Background. Hodgkin lymphoma (HL) is the most common non-AIDS-defining cancer in HIV-positive patients. Studies on South African (SA) populations have described the prevalence as $7-17 \%$ of all lymphomas, $8-27 \%$ of head and neck lymphomas, $9 \%$ of lymph node biopsies and $4 \%$ of HIV-related malignancies.

Objectives. To describe the incidence of HL at our centre between 2005 and 2016 by year, gender, HIV status, histological subclassification and bone marrow involvement, and compare these findings with similar SA and African studies.

Methods. This was a retrospective study of all incident HL cases diagnosed in the Department of Pathology, National Health Laboratory Service, Tygerberg Academic Hospital, Cape Town. Follow-up, relapsed and referral cases were excluded. A positive diagnosis of HL was confirmed by either lymph node or bone marrow biopsy and was based on morphological and immunohistochemical findings in accordance with the World Health Organization classification.

Results. There were 303 incident cases of HL diagnosed. The incidence increased from 2005 to 2011, with a spike in cases in 2008 and a subsequent decline overall after 2011. The highest proportion of cases was in the $25-49$-year-old age category (51.1\%). There were 77 HIV-positive patients (25.4\%), of whom 53 (68.8\%) had CD4+ counts $<500$ cells/ $\mu \mathrm{L}$. In keeping with other African studies, the main subtypes were nodular sclerosis HL (49.8\%) and mixed-cellularity HL (23.1\%). Bone marrow biopsy following lymph node diagnosis of HL confirmed involvement in $23.7 \%$ of patients.

Conclusions. Absolute numbers of cases of HL at our centre have increased since the roll-out of antiretroviral therapy (ART) to the public sector. The recent change in policy to make ART available to all HIV-positive patients independent of CD4+ count suggests that patients will survive longer and are therefore at increased risk of developing HL. We anticipate that numbers of HL cases will increase or remain high in the coming years, and we need to prepare for this.
\end{abstract}

S Afr Med J 2018;108(7):563-567. DOI:10.7196/SAMJ.2018.v108i7.12844

It has been shown in the past 30 years that Hodgkin lymphoma (HL) comprises two different B-cell disease entities, namely classic HL (CHL) and nodular lymphocyte-predominant HL. Although gene expression profiling shows that these two types of HL have similarities, they differ considerably in their morphology, immunophenotype and clinical course. CHL comprises $\sim 95 \%$ of all lymphomas and is further subclassified into four different histological variants, namely nodular sclerosis HL (CHL-NS), mixed-cellularity HL (CHL-MC), lymphocyterich HL (CHL-LR) and lymphocyte-deplete HL (CHL-LD). ${ }^{[1]}$

$\mathrm{HL}$ is the most common non-AIDS-defining cancer in HIVpositive patients, who are at $8-10$ times higher risk than HIVnegative individuals. In 2016, it was estimated that $12.7 \%(7.03$ million) of the population aged 15 - 49 years in South Africa (SA) was HIV-positive, compared with 10.8\% (5.1 million) in 2005.

The global incidence of HL increased by $12.9 \%$ between 2005 and 2015. ${ }^{[2]}$ This rise in incidence largely seems to be linked to HIV infection. Several studies conducted at SA tertiary hospitals have described the prevalence of HL as $7-17 \%$ of all lymphomas, ${ }^{[3-6]}$ $8-27 \%$ of head and neck lymphomas, ${ }^{[7,8]} 9 \%$ of lymph node biopsies and $4 \%$ of HIV-related malignancies ${ }^{[9]}$ presenting at the respective hospitals. HIV seropositivity in these cases ranged between $8 \%$ and
$67 \%{ }^{[3-6,8]}$ The most common subtypes documented were CHL-MC and CHL-NS. ${ }^{[6,8]}$

There are few epidemiological studies of HL in other African countries, with previous studies showing prevalences of HL between $0.5 \%$ and $2 \%$ of all malignancies, ${ }^{[10,11]} 7-12.5 \%$ of all lymphomas ${ }^{[12]}$ and $7-11 \%$ of all lymph node biopsies ${ }^{[13]}$ investigated. HIV seropositivity ranged between $0 \%$ and $16 \%{ }^{[10-14]}$ with some studies not testing for HIV owing to lack of availability of the means to do so. ${ }^{[10,11,13]} \mathrm{A}$ multi-African study ${ }^{[14]}\left(n=52\right.$ cases) and a Nigerian study ${ }^{[1]}(n=80)$ described CHL-MC and CHL-NS as the most common subtypes, whereas studies in Nigeria ${ }^{[13]}(n=56)$ and Malawi ${ }^{[12]}(n=31)$ recorded a predominance of CHL-MC and CHL-LD.

It is reported that HIV-positive patients on antiretroviral therapy (ART) with a higher CD4+ count are at higher risk of developing $\mathrm{CHL}$ than patients with a lower CD4+ count. The risk of developing $\mathrm{HL}$ is highest when the CD4+ count is between 150 and 199 cells/ $\mu \mathrm{L}$. There are several theories regarding the role of ART. One is that ART increases the CD4+ count, which the Hodgkin Reed-Sternberg (HRS) cells are dependent on for their growth. A second theory is that the HRS cell is a crippled B-cell and therefore not directly affected by HIV. As these HRS cells are B-cells they attract CD4+ T-cells, and the 
increase in CD4+ T-cells is therefore a consequence of the tumour cell. A third theory is that there are competing risks, in that patients with lower CD4+ counts are more likely to suffer from AIDs-defining illnesses. ${ }^{[1]}$

As CHL has previously been shown to be dependent on CD4+ counts in HIV-positive patients, it is vital that we establish the baseline incidence and the impact of ART rollout on the incidence of HL in our institution over the past decade. Initiation of ART in the public sector in SA was implemented in patients with a CD4+ count of $<200$ cells $/ \mu \mathrm{L}$ in 2004 . This changed to $<350$ cells $/ \mu \mathrm{L}$ in 2013 and then to $<500$ cells $/ \mu \mathrm{L}$ in 2015. The 2017 Adult Antiretroviral Therapy Guideline recommendation is to treat HIV-positive patients independent of the baseline CD4+ count.

\section{Objectives}

The prevalence of HL at our centre, Tygerberg Academic Hospital (TAH) in the Western Cape Province of SA, was 17\% of all lymphomas diagnosed between 2002 and 2009 ( $n=187$ cases); 8\% of HL patients were HIV-positive. ${ }^{[5]}$ The objective of this study was to expand on this previous prevalence study of all lymphomas conducted at our centre ${ }^{[5]}$ by describing in more detail the incidence of HL between 2005 and 2016 by year, gender, HIV status, histological subclassification and bone marrow involvement, and to compare these findings with other SA and African studies describing HL.

\section{Methods}

A retrospective study of HL cases diagnosed in the Division of Anatomical Pathology and the Division of Haematopathology, Department of Pathology, National Health Laboratory Service (NHLS), TAH, was conducted. TAH is a 1380 -bed tertiary referral academic hospital affiliated to Stellenbosch University and services approximately half of the population of the Western Cape (total population $\sim 6.3$ million). Cases were collected from 1 January 2005 to 31 December 2016 and form part of the ongoing Tygerberg Lymphoma Study Group database (Human Research Ethics Committee ref. no. N07/03/068) established in 2007 in the Division of Haematopathology with the aim of documenting all lymphoma cases presenting at TAH. ${ }^{[5]}$ Patient demographic, HIV test, CD4+ count, lymph node and bone marrow biopsy data for this database were extracted from the DisaLab version 04.16.04.373 (Laboratory System Technology (Pty) Ltd, South Africa) (up to August 2015) and TrakCare v6.10 (InterSystems, Australia) laboratory information systems. Ethical approval of the study was granted by the Health Research Ethics Committee, Stellenbosch University (ref. no. S15/09/206).

\section{Case definition}

A positive diagnosis of HL was confirmed either by lymph node biopsy assessment reported by the Division of Anatomical Pathology or by bone marrow biopsy assessment reported by the Division of Haematopathology. A staging bone marrow biopsy was routinely performed on all lymph node biopsy-positive lymphoma patients at our centre. The lymph node and bone marrow biopsy diagnoses were based on morphological and immunohistochemical findings in accordance with the 2008 World Health Organization classification of tumours of haematopoietic and lymphoid tissues. The bone marrow biopsy procedure and reporting format followed the International Council for Standardisation in Haematology guidelines.

\section{Inclusion and exclusion criteria}

All incident cases of HL of all ages presenting between 1 January 2005 and 31 December 2016 were included in the study. Follow-up, relapsed and referral cases of HL were excluded.

\section{Patient variables}

Demographic characteristics (age and gender), date of diagnosis, HIV status (using enzyme-linked immunosorbent assay HIV serology testing), $\mathrm{CD} 4+$ count (using flow cytometry pan-leucocyte gating techniques), HIV viral load (measured by quantitative RNA-based testing using the Amplicor HIV-1 Monitor Test (Roche Diagnostics, USA)), HL subclassification and presence of bone marrow involvement of HL (stage 4 disease) were collected. Age ranges for analysis were selected as per the 2012 South African National HIV Prevalence, Incidence and Behavioural Survey for comparability.

\section{Data analysis}

Statistical analysis of all data was performed using Excel 2016 (Microsoft, USA) and SPSS v20 (SPSS, USA). Results were summarised and presented by year, gender and HIV status using descriptive statistical methods. As HL is an HIV-related tumour, all variables were analysed by HIV status.

\section{Results}

There were 303 incident cases of HL (56.4\% male and $43.6 \%$ female) diagnosed between 2005 and 2016 (Table 1).

\section{Year}

There was an increasing trend in incidence of cases in both HIV-positive and HIV-negative patients from 2005 to 2011, with a spike in cases in 2008. There was a subsequent overall decline after 2011 (Fig. 1).

\section{Age}

The mean (standard deviation (SD)) age at diagnosis of HL, independent of HIV status, was 33.4 (15.9) years. There was no significant difference in the mean age at diagnosis of HL between the HIV-positive and HIV-negative groups (Table 1). The highest number of cases was found in the 25 - 49-year age range category $(n=155,51.1 \%)$ (Table 1 and Fig. 2).

\section{Gender}

There was no statistically significant difference in gender between the HIV-positive and HIV-negative groups $(p=0.14)$.

\section{HIV status and CD4+ count}

Only $81.2 \%(n=246)$ of our sample had confirmation of HIV testing. There were 77 HIV-positive patients (25.4\%), of whom 64 (21.1\%) had CD4+ counts reported. There were 169 HIV-negative patients (55.8\%) and 57 patients with unknown HIV status (18.8\%). In the HIV-positive group, 43 patients (55.8\%) had a CD4+ count of $<350$ cells $/ \mu \mathrm{L}$ at the time of diagnosis, whereas 53 patients $(71.4 \%)$ had a count of $<500$ cells/ $\mu L$. Only 30 patients $(39.0 \%)$ had a count of $<200$ cells/ $\mu$ l (Table 2 ). The 2015 SA HIV management guidelines recommended changing initiation of treatment in all HIV-positive patients from CD $4+<350$ cells $/ \mu \mathrm{L}$ to $<500$ cells $/ \mu \mathrm{L}$. The mean (SD) CD4+ count at the time of diagnosis for those tested was 225 (173) cells $/ \mu \mathrm{L})$.

\section{Histological subtype}

There were 280 cases of HL (92.4\%) diagnosed by lymph node biopsy. The most frequent subtypes identified were CHL-NS (151 cases, $49.8 \%$ of all subtypes identified) and CHL-MC (70 cases, $23.1 \%$ ) (Fig. 3). Of those patients tested for HIV, more were HIV-negative in these two subtypes (72.2\% v. 67.6\%) (Table 1 and Fig. 3).

\section{Bone marrow involvement (stage 4 disease)}

Bone marrow biopsy following lymph node diagnosis of HL con- 


\begin{tabular}{|c|c|c|c|c|c|}
\hline \multirow[b]{2}{*}{ Characteristics } & \multicolumn{3}{|c|}{ HIV status $(N=303)$} & \multirow[b]{2}{*}{ Total } & \multirow[b]{2}{*}{$p$-value } \\
\hline & $\begin{array}{l}\text { HIV-positive } \\
(n=77,25.4 \%)\end{array}$ & $\begin{array}{l}\text { HIV-negative } \\
(n=169,55.8 \%)\end{array}$ & $\begin{array}{l}\text { Unknown } \\
(n=57,18.8 \%)\end{array}$ & & \\
\hline Age (yr), mean (SD) & $34.4(10.5)$ & $32.2(16.7)$ & $35.6(19.1)$ & & 0.24 \\
\hline \multicolumn{6}{|l|}{ Age (yr), $n(\%)$} \\
\hline $0-14$ & $2(2.6)$ & $21(12.4)$ & $8(14.0)$ & $31(10.2)$ & \\
\hline $15-24$ & $10(13.0)$ & $41(24.3)$ & $8(14.0)$ & $59(19.5)$ & \\
\hline $25-49$ & $59(76.6)$ & $71(42.0)$ & $25(43.9)$ & $155(51.1)$ & \\
\hline $50-59$ & $5(6.5)$ & $25(14.8)$ & $8(14.0)$ & $38(12.5)$ & \\
\hline$\geq 60$ & $1(1.3)$ & $11(6.5)$ & $8(14.8)$ & $20(6.6)$ & \\
\hline Gender, $n(\%)$ & & & & & 0.14 \\
\hline Male & $38(49.4)$ & $101(59.8)$ & $32(56.7)$ & $171(56.4)$ & \\
\hline Female & $39(50.6)$ & $68(40.2)$ & $25(43.9)$ & $132(43.6)$ & \\
\hline \multicolumn{6}{|c|}{ Histological subtype (based on lymph node diagnosis), $n$ (\%) } \\
\hline CHL (no subclassification) & $7(9.1)$ & $20(11.8)$ & $4(7.0)$ & $31(10.2)$ & \\
\hline CHL-NS & $32(41.6)$ & $88(52.1)$ & $31(54.4)$ & $151(49.8)$ & \\
\hline CHL-MC & $20(26.0)$ & $34(20.1)$ & $15(26.3)$ & $69(22.7)$ & \\
\hline CHL-LR & $3(3.9)$ & $6(3.6)$ & 0 & $9(3.0)$ & \\
\hline CHL-LD & $3(3.9)$ & $3(1.8)$ & $3(5.3)$ & $9(3.0)$ & \\
\hline NLPHL & 0 & $8(4.7)$ & $2(3.5)$ & $10(3.3)$ & \\
\hline Inconclusive & $12(15.6)$ & $10(5.9)$ & $2(3.5)$ & $24(7.9)$ & \\
\hline \multicolumn{6}{|c|}{ Bone marrow involvement, $n(\%)$} \\
\hline Yes & $35(45.5)$ & $33(19.5)$ & $4(7.0)$ & $72(23.7)$ & \\
\hline Bone marrow diagnosis & $8(10.3)$ & $5(2.9)$ & $1(1.8)$ & $14(4.6)$ & \\
\hline No & $29(37.7)$ & $121(71.6)$ & $18(31.6)$ & $168(55.4)$ & \\
\hline Not done & $12(15.6)$ & $13(7.7)$ & $35(61.4)$ & $60(19.8)$ & \\
\hline Inadequate bone marrow & $1(1.3)$ & $2(1.2)$ & 0 & $3(1.0)$ & \\
\hline
\end{tabular}

Table 2. Number and percentage of patients in CD4+ categories, 2005 - 2016

\begin{tabular}{lllllll}
\hline & \multicolumn{5}{c}{ CD4+ cell count $($ cells/ $\mu \mathrm{L})$} \\
\cline { 2 - 6 } & $<\mathbf{5 0}$ & $\mathbf{5 1 - 1 4 9}$ & $\mathbf{1 5 0 - 1 9 9}$ & $>\mathbf{2 0 0}$ & Unknown & Total \\
\hline Patients, $n(\%)$ & $10(13.0)$ & $12(15.6)$ & $8(10.4)$ & $34(44.2)$ & $13(16.8)$ & 77
\end{tabular}

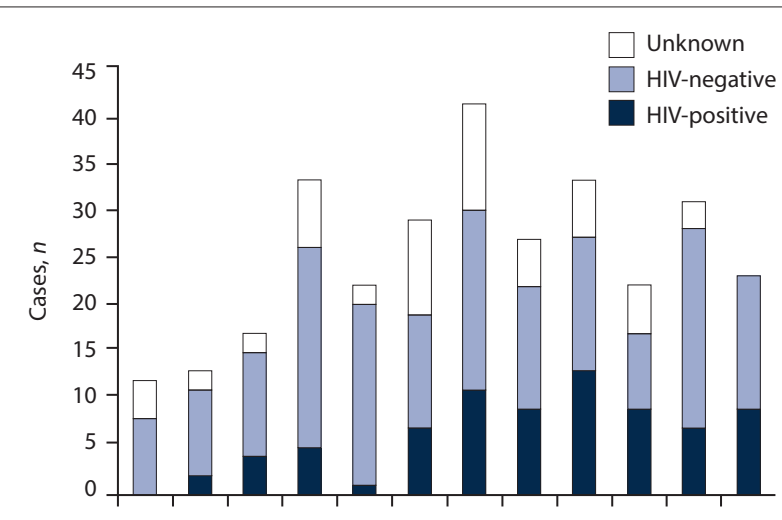

200520062007200820092010201120122013201420152016 Year

Fig. 1. Number of incident Hodgkin lymphoma cases by year and HIV status, 2005 - 2016.

firmed involvement of the bone marrow in 72 patients (23.7\%), while 168 patients (55.4\%) did not have bone marrow involvement (Table 1 and Fig. 4). Of patients with bone marrow involvement, roughly equal numbers were HIV-positive (48.6\%) and HIV-negative

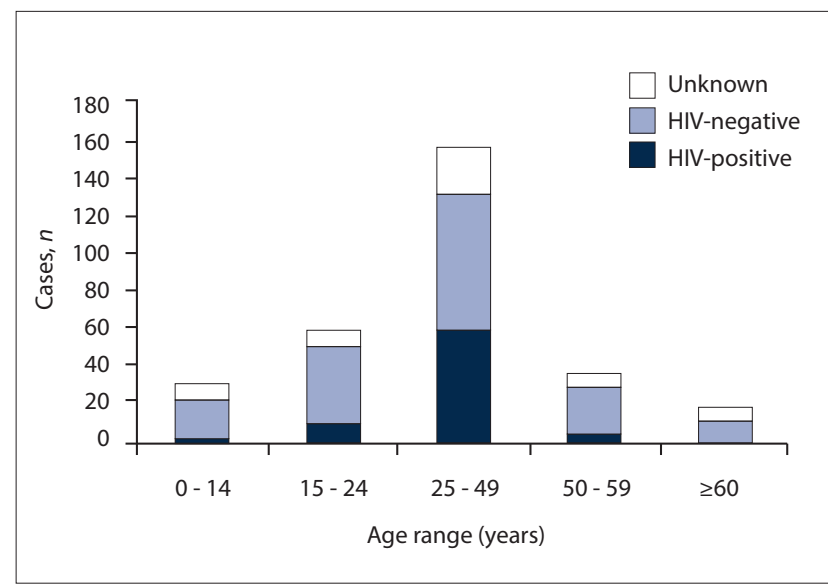

Fig. 2. Age distribution of Hodgkin lymphoma cases by HIV status, 2005 - 2016.

(45.8\%). There were 29 HIV-positive HL patients who did not have bone marrow involvement.

There were 14 cases of $\mathrm{HL}$ that were initially diagnosed from incidental bone marrow biopsies for investigation of pancytopenia or bicytopenia. Subtyping of HL is currently not performed on bone marrow biopsy specimens. 


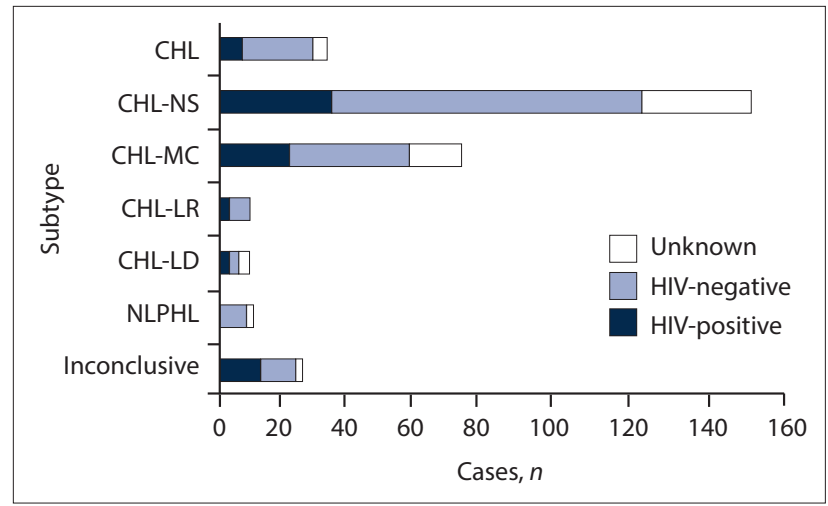

Fig. 3. Hodgkin lymphoma subtype by HIV status (based on lymph node diagnosis), 2005 - 2016. (CHL = classic Hodgkin lymphoma; $C H L-N S=$ nodular sclerosis CHL; CHL-MC = mixed-cellularity CHL; CHL-LR = lymphocyte-rich CHL; CHL-LD = lymphocyte-depleted CHL; NLPHL = nodular lymphocyte-predominant $\mathrm{CHL}$.)

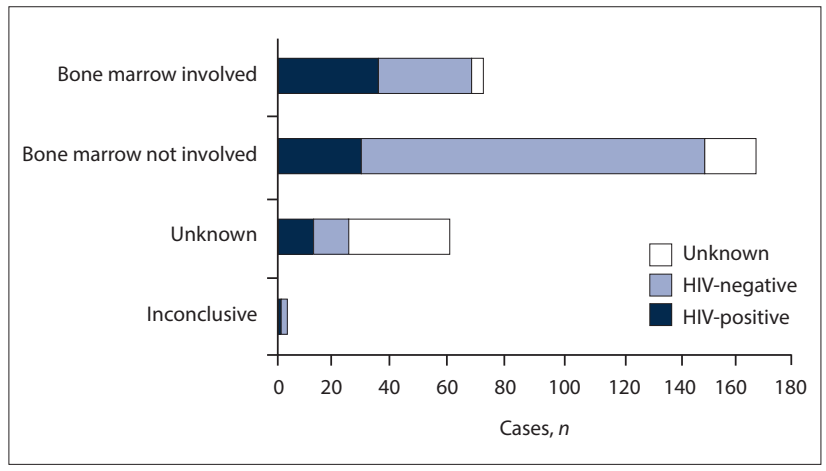

Fig. 4. Bone marrow involvement by HIV status, 2005 - 2016.

\section{Discussion}

Our study identified 303 new cases of HL at Tygerberg Hospital between 2005 and 2016 by lymph node biopsy diagnosis (93.4\%) or bone marrow biopsy diagnosis (4.6\%). HL was not identified in any other extranodal sites.

\section{Increasing trend in HL incidence from 2005 to 2011}

The global incidence of HL increased by $12.9 \%$ between 2005 and $2015 .{ }^{[2]}$ Similarly, a previous study at our institution between 2002 and 2009 showed an increased incidence of all lymphomas, including more rare types such as Burkitt lymphoma and plasmablastic lymphoma, in both HIV-positive and HIV-negative patients. ${ }^{\left[{ }^{[j}\right.}$

The increasing trend in the incidence of HL in our population between 2005 and 2011 is influenced by multifactorial causes. Firstly, ART rollout for HIV-positive patients in the public health sector commenced in 2004. Secondly, urbanisation with migration of people to the Western Cape from other areas of SA and other African countries may have contributed to the increase. International guidelines for the diagnosis and treatment of HL suggest HIV testing as part of risk stratification. Unfortunately, testing for HIV in SA carried a social stigma during the early rise of HIV disease. This resulted in fewer suspected lymphoma patients being tested and therefore could have influenced our data. This is possibly evidenced by the $18.8 \%$ of patients of unknown HIV status in our data, with the majority of these cases before 2012 (66.7\%). Although our study showed that overall more incident cases of HL were HIV-negative, the proportion of patients who were HIV-positive was noted to be on the rise.
Combined ART has been shown to improve survival in HL patients. The total number of patients receiving ART in SA increased from 47500 in mid-2004 to 1.79 million in mid-2011, with $\sim 85 \%$ of these patients receiving ART through the public health sector. ${ }^{[15]}$ There were 5000 patients on ART in the Western Cape in 2004, the figure increasing to 107000 by 2011 in the public, private and nongovernmental organisation sectors combined. The percentage of new patients on ART ranged between 34\% and 64\% each year. From 2004 to 2011, there were twice as many women than men on ART. ${ }^{[15]}$ These increases in ART coverage would be likely to affect the epidemiology of HIV-related lymphomas presenting at our institution.

Early epidemiological studies have suggested a possible association between ART and the incidence of HL. The risk of HL increased by $68 \%$ in HIV-positive patients since ART was introduced in the USA (1996 - 2002) compared with the previous period of no ART (1990 - 1995). ${ }^{[16]}$

\section{HIV status and CD4+ count}

A higher proportion of cases in our study population were HIVnegative $(55.8 \%)$ than HIV-positive (25.4\%). Of the HIV-positive patients, $54.6 \%$ had a CD4+ count $>150$ cells $/ \mu \mathrm{L}$, which is consistent with a US population study of HL from 1980 to 2002 that showed significantly more patients with CD4+ counts of $150-199$ cells/ $\mu \mathrm{L}$ compared with $<50$ cells $/ \mu \mathrm{L} .{ }^{[17]}$ None of the studies on HL in African populations reviewed had documented CD4+ counts.

A study of several US states linking HIV/AIDS and cancer registry data in patients to identify HL cases (1980 - 2002, $n=173$ cases) concluded that patients with moderate levels of immunodeficiency (CD4+ count $150-199$ cells/ $\mu \mathrm{L})$ were at higher risk of developing HL than those with more severe levels of immunodeficiency (CD4+ count $<50$ cells $/ \mu \mathrm{L}$ ). Furthermore, in another study the mean $\mathrm{CD} 4+$ count at diagnosis of HL was 210 cells $/ \mu \mathrm{L}$, which was found to be relatively high compared with other HIV-associated non-HL. ${ }^{[18]}$ Our study found that $\sim 55 \%$ of HIV-positive HL patients had a CD4+ count $>150$ cells $/ \mu \mathrm{L}$, in keeping with the US study. This finding further supports the hypothesis that the tumour microenvironment (non-neoplastic reactive cells) releases feedback cytokines and chemokines that stimulate the proliferation or inhibition of apoptosis of HRS cells. ${ }^{[18]}$

However, a subsequent Swiss cohort of HIV-positive patients followed up for 20 years $(n=14606)$ concluded there was no evidence of increased HL risk with ART and improved immunity. A study in Uganda (1999 - 2008) evaluated the effect of ART on HIV-related cancer incidence and further concluded that there was no association between increased ART coverage and incidence of $\mathrm{HL}$ ( $n=153$ cases). ${ }^{[19]}$

\section{Age and gender}

HIV seroprevalence in this study population was highest in the 25 49 -year age group (76.6\%), which is also the age group most affected by HIV in SA. There was no difference between male and female patients in our study, which is in keeping with a previous study in several sub-Saharan African countries ${ }^{[14]}$ and in Western populations.

\section{Decreasing trend from 2012 to 2016}

Although the trend from 2012 to 2016 differs from that for 2005 to 2011, absolute numbers of HL have increased since 2005. Further investigation is necessary to identify the underlying causes for these differences during this period.

\section{Histological subtype}

The predominant subtypes (CHL-MC and CHL-NS) in HIV- 
positive patients are in keeping with previous SA studies ${ }^{[-8]}$ and with two African studies. ${ }^{[1,14]}$ Two other African studies showed a predominance of CHL-MC and CHL-LD. ${ }^{[12,13]}$ In contrast, the most common HL subtypes in Western populations are CHL-MC and CHL-LD.

\section{Bone marrow involvement (stage 4 disease)}

Our HL population included 72 cases $(23.7 \%)$ with bone marrow involvement, compared with 6 cases $(50 \%)$ in a recent Malawian study. ${ }^{[12]}$ Of these 72 patients, $14(19.4 \%)$ were initially diagnosed by bone marrow biopsy. This finding supports clinical observations that HIV-positive patients with lymphoma may present atypically. In our setting, the diagnosis of lymphoma may be delayed owing to the increase of concomitant TB and HIV, as the clinical presentations overlap. HIV patients may therefore present with extensive disease. This is an important finding, and we recommend that clinicians have a high index of suspicion when HIV-positive patients present with suggestive clinical features, namely B-cell symptoms.

\section{Study strengths and limitations}

A strength of our study was the long period of observation and the comprehensive laboratory information management systems at TAH. Limitations include the retrospective study design and incomplete data on the HIV status of $18.8 \%$ of the cohort. Further, we did not have data on ART use in this cohort. We also did not have data on patients diagnosed at other centres/departments or in the private health sector and then subsequently referred to TBH for treatment.

\section{Conclusions}

Our study showed that the absolute numbers of HL at our centre increased over the 10 years after roll-out of ART to the public sector. The change in recent policy of ART availability to all HIV-positive patients independent of CD4+ count suggests that patients will survive longer and are therefore at increased risk of developing HL. Based on the study findings, we anticipate that numbers of HL cases will increase or remain high in the coming years, and we need to increase awareness and to prepare for this. Finally, our findings highlight the value of a regional and national cancer registry linked to an HIV test result database for the monitoring of HIV-related malignancies such as HL.

Acknowledgements. We thank the NHLS at Tygerberg Hospital for use of their database and Mr W Kleynhans (IT Department) for data access and retrieval.
Author contributions. NN contributed to the study design, analysis and manuscript writing. RG contributed to conceptualisation, study design and manuscript writing. AA and CL contributed to critical review. FM contributed to data collection and analysis.

Funding. We thank the Cancer Association of South Africa for their financial contribution towards this study.

Conflicts of interest. None. The views expressed in this article are the authors own and not the official position of the NHLS and Stellenbosch University.

1. Martis N, Mounier N. Hodgkin lymphoma in patients with HIV infection: A review. Curr Hemato Malig Rep 2012;7(3):228-234. https://doi.org/10.1007/s11899-012-0125-2 2. Fitzmaurice C, Allen C, Barber RM, et al. Global, regional, and national cancer incidence, mortality,
years of life lost, years lived with disability, and disability-adjusted life-years for 32 cancer groups, 1990 years of life lost, years lived with disability, and disability-adjusted life-years for 32 cancer groups, 1990
to 2015: A systematic analysis for the Global Burden of Disease Study. JAMA Oncol 2017;3(4):524-548. to $2015:$ A systematic analysis for the Global Bu
https://oi.org/10.1001/jamaoncol.2016.5688

3. Patel M, Philip V, Omar T, et al. The impact of human immunodeficiency virus infection (HIV) on Patel M, Philip V, Omar T, et al. The impact of human immunodeficiency virus infection (HIV) on Wigell TM, Matin $\mathrm{H}$, Willem $\mathrm{P}$ et al Changing Wiggill TM, Mantina H, Willem P, et al. Changing pattern of lymphoma subgroups at a tertiar academic complex in a high-prevalence HIV setting: A South African perspective. J Acquir Immune
Defic Syndr 2011;56(5):460-466. https://doi.org/10.1097/QAI.0b013e31820bb06a

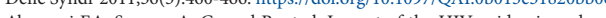

5. Abayomi EA, Somers A, Grewal R, et al. Impact of the HIV epidemic and anti-retroviral treatment policy on lymphoma incidence and subtypes seen in the Western Cape of South Africa, 2002 - 2009 Preliminary findings of the Tygerberg Lymphoma Study Group. Transfus Apher Sci 2011;44(2):161 166. https://doi.org/10.1016/j.transci.2011.01.007

6. Mantina H, Wiggill TM, Carmona S, et al. Characterization of lymphomas in a high prevalence HIV setting. J Acquir Immune Defic Syndr 2010;53(5):656-660. https://insights.ovid.com/ pubmed?pmid $=20160652$ (accessed 28 May 2018)

7. Alli N, Meer S. Head and neck lymphomas: A 20-year review in an oral pathology unit, Johannesburg, South Africa, a country with the highest global incidence of HIV/AIDS. Oral Oncol 2017;67(4):17-23. https://doi.org/10.1016/.joraloncology.2017.01.011

8. Chetty M, Sudi S, Abayomi EA. Prevalence and spectrum of head and neck lymphomas at Tygerberg Hospital, South Africa, 2003 to 2007. J S Afr Dent Assoc 2012;67(6):270-277. http://journals.co.za/ content/sada/67/6/EJC143694 (accessed 28 May 2018).

9. Reddy DL, Venter WD, Pather S. Patterns of lymph node pathology; fine needle aspiration biopsy as an evaluation tool for lymphadenopathy: A retrospective descriptive study conducted at the largest hospital in Africa. PloS One 2015;10(6):1-10. https://doi.org/10.1371/journal.pone.0130148

10. Chokunonga E, Borok MZ, Chirenje ZM, et al. Trends in the incidence of cancer in the black population of Harare, Zimbabwe 1991 - 2010. Int J Cancer 2013;133(3):721-729. https://doi.org/10.1002/ijc.28063

1. Oluwasola AO, Olanivi JA, Otegbayo JA, et al. A fifteen-year review of lymphomas in a Nigerian tertiary healthcare centre. J Health Popul Nutr 2011;29(4):310-316. https://www.ncbi.nlm.nih.gov/ pmc/articles/PMC3190361/

12. Westmoreland KD, Stanley CC, Montgomery ND, et al. Hodgkin lymphoma, HIV, and Epstein-Barr virus in Malawi: Longitudinal results from the Kamuzu Central Hospital Lymphoma study. Pediatr Blood Cancer 2017;64(5):e26302. https://doi.org/10.1002/pbc.26302

13. Olu-Eddo AN, Omoti CE. Hodgkin lymphoma: Clinicopathologic features in Benin City, Nigeria and update on its biology and classification. Niger J Clin Pract 2011;14(4):440-444. https://doi. and update on its biology and
org $/ 10.4103 / 1119-3077.91752$

14. Naresh KN, Raphael M, Ayers L, et al. Lymphomas in sub-Saharan Africa - what can we learn and how can we help in improving diagnosis, managing patients and fostering translational research? $\mathrm{Br}$. Haematol 2011:154(6):696-703. https://doi.org/10.1111/.1365-2141.2011.08772.x

15. Johnson LF. Access to antiretroviral treatment in South Africa, 2004 - 2011. South Afr J HIV Med 2012;13(1):22-27. https://doi.org/10.1080/09540121.2014.994471

16. Engels EA, Pfeiffer RM, Goedert JJ, et al. Trends in cancer risk among people with AIDS in the United States 1980 - 2002. AIDS 2006;20(12):1645-1654. https://doi.org/10.1097/01.aids.0000238411.75324.5 7. Biggar RJ, Jaffe ES, Goedert JJ, et al. Hodgkin lymphoma and immunodeficiency in persons with HIV AIDS. Blood 2006;108(12):3786-3791. https://doi.org/10.1182/blood-2006-05-024109

18. Carbone A, Vaccher E, Gloghini A, et al. Diagnosis and management of lymphomas and other cancers in HIV-infected patients. Nat Rev Clin Oncol 2014;11(4):223-238. https://doi.org/10.1038 nrclinonc.2014.3

19. Mutyaba I, Phipps W, Krantz EM, et al. A population-level evaluation of the effect of antiretrovira therapy on cancer incidence in Kyadondo County, Uganda, 1999 - 2008. J Acquir Immune Defic Syndr 2015;69(4):481-486. https://doi.org/10.1097/QAI.0000000000000620

Accepted 13 February 2018 